\title{
EL PINTOR FELIPE ABÁS, DISCÍPULO DE GOYA
}

\author{
POR \\ WIFREDO RINCÓN GARCÍA \\ Departamento de Historia del Arte, CSIC
}

The author provides new data - biographic as well as of artistic significance - on Aragonese painter Felipe Abás, a disciple of Goya's. He also discusses a signed painting of his, unknown to this date.

Manuel Ossorio y Bernard, en su Galería Biográfica de artistas españoles del siglo XIX, publicada en su primera edición en 1868 y en nueva y segunda edición ampliada en 1884, incluye al pintor Felipe Abás, del que comienza su biografía — de notable contenido- con la apreciación de que se trata de un «pintor de historia», debiéndose entender esta calificativo no en el sentido decimonónico sino en el dieciochesco, como pintor de composiciones. Esta breve biografía es prácticamente el único soporte con el que nos enfrentamos a la hora de elaborar este artículo con el que pretendemos recuperar la figura y obra de este pintor aragonés, uno de los escasos discípulos del genial Francisco de Goya, del que afirmó Sánchez Cantón que «si es artista, no ha pasado a los diccionarios» ${ }^{1}$

\section{Nacimiento y formación en la Real Academia de Bellas Artes de San Luis de Zaragoza}

Conocemos la fecha de nacimiento de Felipe Abás por la partida de bautismo existente en la iglesia parroquial de Nuestra Señora de la Asunción de Calaceite (Teruel), su localidad natal: «Dia uno de Mayo de mil setecientos setenta y siete yo el infra firmado bautice segun rito de N. M. la Yglesia a Felipe Abas, hijo legitimo y natural de Manuel Abas y de Francisca Aranda consortes de esta: nació el día antes a las tres de la tarde fueron Padrinos Francisco Abás y Maria Monreal a quienes esplique el parentesco y la obligación

\footnotetext{
${ }^{1}$ Sánchez Cantón, 1946, p. 80, nota 1.
} 
de instruir el niño y para que conste lo firmo dicho dia, mes y año. Fran ${ }^{\text {co }}$ Xavier Martín $P^{c o}$ Vicario» ${ }^{2}$

Ya había precisado la fecha de su nacimiento, el 30 de abril de 1777, Ossorio y Bernard ${ }^{3}$, completando esta información Santiago Vidiella, quien en 1896 llevaba a cabo la publicación de su trabajo Recitaciones de la Historia Política y Eclesiástica de Calaceite. Aunque en esta edición no recoge ninguna información sobre Abás, el autor siguió trabajando, hasta su muerte acaecida en 1929, en la ampliación de su trabajo inicial con la redacción de distintos Apéndices, figurando en el 140: «Puedo aquí dar noticia de otros ilustres hijos de nuestra villa adquirida después de editar las Recitaciones», apéndices éstos que se han incluido, en su lugar correspondiente, en la nueva edición de esta libro llevada a cabo un siglo más tarde, en 1996, a cargo del Ayuntamiento de Calaceite, del Instituto de Estudios Turolenses, Centro de Estudios Bajoaragoneses y Familia Jassa, propietaria de los manuscritos.

Vidiella pone de manifiesto al tratar de la biografía de Felipe Abás Aranda: «Tomo esta nota de la Galería Biográfica de artistas españoles del siglo XIX (2. . edición ampliada, Madrid, 188384) por M. Osorio y Bernard» ${ }^{4}$, indicando que «a lo dicho por Osorio puedo añadir que D. Felipe fue tercer hijo del matrimonio contraido en 1770 por Manuel Abás y Francisca Aranda; que fue bautizado en $1 .^{\circ}$ de Mayo del citado año 1777, y que la abuela materna del pintor llamose Orosia, lo cual explica el proyecto de trabajar el cuadro de la Santa para nuestra parroquial» ${ }^{5}$.

El mismo Vidiella, en el capítulo VI de sus Recitaciones, que titula «Correría genealógica», se ocupa del apellido Abás: «Los de Abás hacían venir su origen (bien que entre los genealogistas reinaba en este punto gran discordancia) del solar de igual nombre en las montañas de Jaca, donde un esforzado militar llamado Fortún, al servicio del rey D. Sancho Ramírez, puso el primero su planta en el castillo de Abás, mató a su alcaide moro y contribuyó, en una palabra, con gran valentía a la toma de la fortaleza. En memoria de la hazaña adoptó para los suyos el sobrenombre que nos ocupa de tanto sabor morisco. Dilatose la descendencia al paso que se extendía por los pueblos de Aragón. En éste [Calaceite] Manuel Abás casaba en 1540 con Isabel Serrano, en la cual procreó a Juan Abás, quien a su vez contrajo matrimonio con Juana García y Medina, natural e hidalga de Cretas; y no fue menospreciado el calaceitano en esta alianza con los nobles Garcías del vecino lugar, porque su familia conservaba la calidad infanzona traída del solar originario de los Abases. Miguel, hijo de aquellos, hizo llegar a Valencia una rama del apellido, que aún a fines de la pasada centuria [siglo XVIII] subsistía allí con nobleza reconocida» ${ }^{6}$.

Pocos datos conocemos de esta familia Abás en el siglo XVIII, recogiendo Vidiella algunos otros miembros de la familia del siglo XVII, varios de ellos eclesiásticos y destacando que en la mañana del día 17 de mayo de 1753 cinco ancianos elevaban la primera piedra de la nueva torre de la iglesia parroquial, repitiéndose la escena en la tarde del mismo día, cuando otros cinco ancianos, los de mayor edad de Calaceite —entre los que se encontraba Tomás Abás, de 90 años, tal vez bisabuelo de nuestro artista - elevaban la segunda de la piedras ${ }^{7}$ y un año más tarde, en 1754, cuando los habitantes de la Cuesta tuvieron la idea de levantar una capilla a la Virgen del Pla, devoción de gran arraigo en Calaceite, «Ante los moradores de la plazuela y sus aledaños,

\footnotetext{
${ }^{2}$ Archivo Parroquial de Nuestra Señora de la Asunción, Calaceite, tomo $2 .^{\circ}$ de bautizados, fol. 391 . Agradezco esta partida de bautismo a doña Teresa Jassá, de Calaceite.

3 Ansón Navarro en su trabajo Academicismo y enseñanza de las Bellas Artes en Zaragoza durante el siglo XVIII. Precedentes, Fundación y Organización de la Real Academia de Bellas Artes de San Luis, publicado en Zaragoza en 1993, incluye una breve biografía de Abás entre los primeros alumnos de la Escuela de Dibujo y de la Academia (1784-1800), dando como fecha de nacimiento la de 1782, totalmente incorrecta de acuerdo con la documentación existente.

4 Edición ya mencionada de 1996, p. 400.

5 Edición de 1996, p. 401.

${ }^{6}$ Edición de 1996, p. 410.

${ }^{7}$ Edición de 1996, p. 351.
} 
gozaban honroso predicamento por aquellos días los labradores Francisco Abás y Jaime Fontcuberta, quienes fueron, no solo los despertadores del proyecto, sino principales pagadores, $y$, por sufragio unánime del barrio, directores de la obra encargados de llevarla pronto y bien a feliz término» ${ }^{8}$. Este Francisco Abás bien pudiera ser el abuelo del pintor.

En 1793 ingresaba como uno de sus primeros alumnos en la Real Academia de Bellas Artes de San Luis de Zaragoza, fundada un año antes, por Real Decreto del Rey Carlos IV de fecha 17 de abril de 1792.

Queriendo la Academia evaluar los progresos de sus primeros alumnos, a finales del año 1796 se encargó a los Académicos de Honor Antonio Ranz Romanillos y José Broto la redacción de un informe, del que se conserva el original dirigido al Presidente, en el archivo de la Academia en el legajo correspondiente a 1796.

Su contenido es de notable interés y pone de manifiesto algunos interesantes aspectos de la vida académica en los años en que Abás era alumno de la academia zaragozana, por lo que transcribimos su contenido:

«Ex ${ }^{\mathrm{mo}}$. Señor. Para desempeñar el encargo que V. Ex. se sirvió hacernos de informar sobre el estado y progresos de los Alumnos de la Academia en cada una de las Artes, hemos oido a sus distinguidos profesores los SS. La Cruz, Ypas, Ynchauste y Rocha, y si bien no hemos dexado de concevir felizes esperanzas para lo venidero, quando las lecciones de estos Maestros hayan adiestrado mas su mano, y secundado mas su imaginacion; en el dia segun el juicio de los mismos profesores en ninguna Arte se hallan los Alumnos con los principios necesarios, y menos con el exercicio y soltura que son indispensables para aspirar a hacer ni siquiera una cosa mediana, y que no sea un miserable borron entregados a su invencion propia. Así es preciso decaer de la esperanza de proponer asuntos grandes y dignos para los premios que la Academia meditaba, por que nadie se presentaría a pretenderlos y seria comprometer en algún modo, en nuestro concepto, la opinion de la Academia el llamar con la proposicion de los premios la expectacion del Publico y dexarla despues frustrada. El que concurriesen aspirantes de otras Provincias no llenaria el objeto de alimentar la emulacion de nuestros jovenes, y acaso les serviria de desaliento: fuera de que lo mas probable seria que no se presentarse aspirante alguno, y en la solemnidad de la Junta publica no hubiese venzedores que coronar y faltase enteramente el motivo de celebrarla; lo que es muy para tenerse en consideración. Proponer asuntos mas faciles y acomodados a la disposicion de unos principiantes y premiar su mediano desempeño con grande pompa y aparato, tampoco se cree decoroso ni correspondiente a lo mucho que en la oponión común promete una Junta Publica de la Academia destinada a la distribucion de premios. Somos pues de dictamen, y asi lo proponemos a esta Junta (o si pareciere mas conforme a los Estatutos) o a la Junta particular, que por ahora y hasta $\mathrm{q}^{\mathrm{e}}$ con pequeños ensayos se vaya conduciendo a los Alumnos de la Academia por pasos graduados a cosas mas elevadas y dignas de la vista publica, se suspenda la proposicion de premios que hayan de distribuirse con la celebridad que se habia pensado. Mas con todo esto por si la Academia inclinase sin embargo de lo expuesto a la opinion de que contando con lo que puedan desempeñar los Discipulos mas adelantados de esta Escuela, se les propongan proporcionados asuntos ofreciendo premiar a los que se aventagen en su execucion oidos y consultados los profesores, juzgamos que los asuntos podrán ser solo, sin elevarse a mas por ahora y sin asignar más que un premio en cada clase los siguientes=

Pintura. Primera Clase.

Copiar baxo la direccion del Maestro un Quadro de una o dos figuras que tenga alguna parte de desnudo, el que el mismo Maestro y los Academicos que tenga a bien la Junta elijan, ya sea de la Academia misma o ya de algun particular aficionado...

\footnotetext{
${ }^{8}$ Edición de 1996, p. 367.
} 
Al desempeño de estos asuntos informan los profesores que podrán concurrir algunos de sus discípulos en cada Arte y así podra lograrse el fin de desempeñarlos con este motivo a la practica de los principios que han aprehendido que no es el menos principal de la distribucion de premios y el de excitar entre ellos una noble emulación que es para el adelantamiento el mas poderosos incentivo. Y aun para esto conceptuamos que contribuiria más la asignacion de premios modicos distribuidos en cada trimestre de la temporada de enseñanza que no los premios de aparato destribuidos de tres en tres años, especialmente mientras los Alumnos se están formando y llega el dia de que puedan dar dignas muestras de su ingenio. Este concepto que hemos formado lo sujetamos a la decision de la Academia, que con el mayor conjunto de luzes, acordará lo que sea mas conveniente para el logro de la mejor enseñanza publica y su mayor lustre».

Tras celebrarse Junta Ordinaria, se contemplaba este tema en la Junta Particular de 23 de diciembre, conteniéndose los temas tratados en un certificación de la misma existente en el archivo: «En Junta Particular de 23 de Dic ${ }^{\text {re }}$ de 1796 a q asistieron los SS. D Juan Martin de Goicoechea VicePresid $^{\text {te }}, \mathrm{D}^{\mathrm{n}}$ Jorge del Rio, Consiliario, y el Secret ${ }^{\mathrm{o}}$ leido el acuerdo anteced ${ }^{\text {te }}$ se vio el papel formado por los SS. $\mathrm{D}^{\mathrm{n}}$ Antonio Ranz Romanillos y $\mathrm{D}^{\mathrm{n}}$ Josef Broto acerca de los Premios $q^{\mathrm{e}}$ podrán ser acomodados a la calidad de los Discipulos $\mathrm{q}^{\mathrm{e}}$ ha remitido la Junta $\operatorname{Ord}^{\mathrm{a}}$ a la Particular para $\mathrm{q}^{\mathrm{e}}$ vea los premios $\mathrm{q}^{\mathrm{e}}$ se pueden distribuir por la $\mathrm{G}^{\mathrm{l}}$ y diga su dictamen $\mathrm{p}^{\mathrm{a}} \mathrm{q}^{\mathrm{e}}$ la $\mathrm{Ord}^{\mathrm{a}}$ arregle los puntos facultativos, y examinada largam ${ }^{\mathrm{te}}$ la materia, acordó la Junta Particular, $\mathrm{q}^{\mathrm{e}}$ al fin de la actual temporada de estudios se podrían distribuir entre los Discipulos concurrentes a la Academia los premios siguientes.

3 De a $300 r^{s}$ a los $3 q^{e}$ mejor desempeñen los asuntos en la $1^{\text {a }}$ Clase de Pintura, Escultura y Arquitectura... $900 \mathrm{r}^{\mathrm{s}}$.

3 De a doscientos $r^{s}$. a los $\mathrm{q}^{\mathrm{e}}$ les sigan en la misma $1^{\mathrm{a}}$ clase... $600 \mathrm{r}^{\mathrm{s}}$. ....

Habiendose conformado la Junta Particular con la adjudicacion de estas cantidades, resolvió $\mathrm{q}^{\mathrm{e}}$ al fin de la temporada actual de estudios se distribuyan por la Junta $\mathrm{G}^{1}$ los premios arriba expresados, y que siendo privativo de la ordin ${ }^{\mathrm{a}}$ arreglar todos los puntos facultativos se de cuenta en ella de esta resolucion $\mathrm{p}^{\mathrm{a}} \mathrm{q}^{\mathrm{e}}$ en la misma se acuerden los asuntos $\mathrm{q}^{\mathrm{e}}$ han de trabajar los discipulos, los plazos $\mathrm{q}^{\mathrm{e}}$ se les han de dar $\mathrm{y}$ todos las circunstancias y calidades $\mathrm{q}^{\mathrm{e}}$ hayan de tener las obras...» ${ }^{9}$.

Para concretar estos aspectos redactaban Jose Broto y Antonio Ranz Romanillos un informe que con fecha de 5 de febrero de 1797 hacían llegar al Presidente de la docta corporación, conteniendo los extremos de la convocatoria: «Ex ${ }^{\mathrm{mo}}$ Señor. Habiendo resuelto V. Ex. en Junta particular que entre los Discipulos de la Academia al fin de la actual temporada de Estudios diez y ocho premios en la forma y cantidad que igualmente se ha servido acordar; para que esta distribución tenga efecto segun las intenciones y miras que la Academia tiene manifestadas, hemos creido, comsultados los Profesores de las tres Nobles Artes, que los asuntos que pueden proponerse a los Discípulos que quieran aspirar al logro de los premios señalados pueden ser en cada una de las Artes los siguientes:

Pintura primera Clase. Copiar bajo la direccion del Maestro $\mathrm{D}^{\mathrm{n}}$ Alexandro Lacruz, el Quadro del Samaritano, obra del celebre Michael Angelo que posee el Ylt ${ }^{\mathrm{mo}}$ Cabildo de esta $\mathrm{S}^{\text {ta }}$. Yglesia Metropolitana y se ha servido franquearlo generosamente a la Academia para este fin, zeloso por los progresos de sus Alumnos... Las Condiciones que pueden prescribirse a los Aspirantes a los premios son las siguientes $=$ Que los opositores hayan de ser precisamente Alumnos de esta $\mathbf{R}^{1}$ Academia de $\mathrm{S}^{\mathrm{n}}$ Luis de los que actualmente concurren y se instruyen en ella en lo que se estára a las Listas e informes de los Directores, no admitiéndose de ningún modo a los que estos no

\footnotetext{
${ }^{9}$ Archivo de la Real Academia de Nobles y Bellas Artes de San Luis de Zaragoza. Libro de Resoluciones de la Junta Particular de la Real Academia de San Luis, 1793-1817, fols. 91 v.-92 v.
} 
reconozcan por tales Alumnos. Que los concurrentes hasta el día 20 de este Mes que se señala por término preciso, se hayan de presentar en la Secretaría de la Academia a declarar el Arte y clase en que aspiran a ser Opositores, firmando en las Listas que con separación de artes y clases se formarán en aquella oficina: Que hecha esta diligencia se han de presentar a los Directores de las respectivas Artes para trabajar bajo su direccion y dexar siempre en su poder y a su custodia las obras para evitar que en los concurrentes se premien agenos trabajos. Que concluidas las obras acompañados de su Maestro y Director las hayan de presentar en la Secretaria o depositar con asistencia del $S^{\text {or }}$ Academico Secretario en la Pieza que se destinare, señaladas con la cifra o numero, sello, que tubieren por conveniente; del que quedará razón en la Secretaria, para que no viendose en las mimas Obras los nombres de sus autores puedan juzgarse con mas imparcialidad. Y finalmente que a lo más tardar hayan de estas entregadas las obras en la forma antes prevenida el día 15 de Mayo de este año. Las quales Condiciones se anunciarán a los Alumnos de la Academia por Edictos que se fixaran inmediatamente en las puertas de las Escuelas y aun los Maestros mismos podran enterar de ellas a los Discipulos que crean en estado de mostrarse Opositores. Pasado el dia 15 de Mayo el $S^{\text {or }}$ Presidente en el que tenga por más oportuno podrá convocar a Junta General para la graduación y juicio de las obras y asignacion de los premios, entendiendose que habiendose de dar dos premios en cada clase, el primero se adjudicara a la Obra que tubiere mas votos en su favor, y el segundo a la que en el numero de votos la siguiere inmediatamente, y si sucediere reunir una todos los votos o hallarse despues dos o más que tengan igual numero en su favor, se procederá a la votación separada del segundo premio. Si esta operacion se hallase executada para el dia de $S^{n}$ Fernando, en el podría celebrarse la otra Junta General de la adjudicacion y entrega de los premios a los que los hubieren ganado. La Academia, a cuyo juicio se sugetan estas ideas podrá rectificarlas con sus superiores luzes como estime más conveniente y de mayor adelantamiento para sus Alumnos, que es a lo que dirige todas sus tareas» ${ }^{10}$.

Aprobados en la Junta Ordinaria del mismo día 5 de febrero los asuntos para los premios, al día siguiente se colocaban los Edictos con los temas y las condiciones que hemos visto en el documento anterior, conservándose un borrador o Edicto manuscrito en el legajo correspondiente a ese año en el archivo académico. En este documento se determinan también las cantidades asignadas para cada una de las clases: «Los Premios que se distribuiran son los $\mathrm{q}^{\mathrm{e}}$ siguen. Tres de a trescientos $r^{s}$ a los tres que mejor desempeñen los asuntos de la $1 .^{a}$ clase de Pintura, Escultura y Arquitectura».

En el expediente correspondiente a este concurso, figura un documento titulado «Lista de los Discipulos $\mathrm{q}^{\mathrm{e}}$ firman a las opisiciones de los Premios ofrecidos por la $\mathrm{R}^{1} \mathrm{Acad}^{\mathrm{a}}$ de $\mathrm{S}^{\mathrm{n}}$ Luis. Pintura. Primera Clase», donde figuran las firmas de los cuatro alumnos que concurrieron antes de la fecha establecida de 20 de febrero: «Felipe Abás, Josef Dordal, Tiburcio López y Ramón Urquizo», poniéndose aquí de manifiesto el desarrollo del concurso, pues junto a las firmas de los dos que concurrieron, Abás y Urquizu, figura un signo en el lado izquierdo y en el derecho una + y las letras L y M, iniciales con que fueron marcadas las obras de ambos artistas. Debajo de estas cuatro firmas, el desarrollo de la votación, apareciendo 6 votos para Abás y ninguno para Urquizu. También se conserva en el mismo expediente otro documento titulado «Lista de los opositores a los Premios ofrecidos por la Real Academia de $S^{n}$ Luis», donde se pone de manifiesto a los dos únicos concurrentes por la Primera Clase de Pintura:

«Felipe Abas... cuya obra esta señalada con la $L^{\text {tra }} L$

Ramon Urquizu cuya obra esta señalada con la Letra M».

El reparto de los premios tuvo lugar el día 25 de agosto de 1797, cuando la Real Academia

${ }^{10}$ Archivo de la Real Academia de Nobles y Bellas Artes de San Luis de Zaragoza. Legajo correspondiente a 1797. 
celebraba la fiesta onomástica de la soberana, la reina doña María Luisa de Parma, por la que había tomado el nombre de San Luis Rey de Francia, constando su desarrollo en un acta de la misma sesión:

«Zaragoza 25 de Agosto de 1797.

La $\mathrm{R}^{1}$ Academia de $S^{\mathrm{n}}$ Luis en celebridad del augusto nombre de la Reyna nuestra Sra. distribuyó en Junta Gen ${ }^{1}$ de hoy los premios particulares de estimulo $q^{\mathrm{e}}$ tenia ofrecidos a sus Alumnos en la forma siguiente:

Pintura. A $D^{n}$ Felipe Abas $300 \mathrm{r}^{s}$. $\mathrm{v}^{\mathrm{n}}$ por el primer premio de la primera clase de Pintura, cuyo asunto fue copiar el quadro del Samaritano, obra del celebre Michael Angelo y $200 \mathrm{r}^{\mathrm{s}}$ a $\mathrm{D}^{\mathrm{n}}$ Ramon Urquizu que obtuvo el Segundo Premio del mismo asunto...

Presidio este acto el Vice-Presid ${ }^{\text {te }}$ de la Academia $\mathrm{D}^{\mathrm{n}}$ Juan Martin de Goicoechea, Caballero de la $\mathrm{R}^{1}$. y distinguida Orden Española de Carlos $3 .^{\circ}$, concurriendo bastante num ${ }^{\circ}$ de Consiliarios, Academicos de Honor, Profesores, y muchos Socios, e individuos de la Rl. Sociedad Arag $^{\text {sa }}$ quedando todos con la mayor satisfaccion de los Alumnos en los primeros años de esta util establecim ${ }^{\text {to }}$. Dio el Vice-Presid ${ }^{\text {te }}$ las gracias a los Maestros por su esmero y cuidado y concluyo con un razonam ${ }^{\text {to }}$, dirigido a manifestar las utilidades de esta enseñanza publica y gratuita, y estimular la aplicacion de los Alumnos».

Otro documento - también del mismo archivo académico - nos pone de manifiesto un nuevo dato sobre las obras de Abás y Urquizu, pues tras exponer que estos dos pintores «copiaron el quadro del Samaritano, obra del celebre Michael Angelo que posehe el $\mathrm{Ytt}^{\mathrm{mo}}$ Cavildo de esta Santa Yglesia Metropolitana», se dice en él que "por algunas diferencias, copiaron despues la cabeza del herido del propio Quadro, y obtuvo el primer Premio de $300 \mathrm{r}^{\mathrm{s}}$ Felipe Abas y el $2 .^{\circ} \mathrm{de}$ $200 \mathrm{r}^{s}$ Ramon Urquizu». El cuadro, según Ossorio, se guardaba en el Museo provincial de Zaragoza $^{11}$.

Años mas tarde, en Junta Particular celebrada el día 1 de febrero de 1805, cuando Felipe Abás culminaba su carrera de formación en la Real Academia de San Fernando, la Real Academia de Bellas Artes de San Luis de Zaragoza acordaba distinguirle, recogiéndose así en el acta de la sesión: «Habiendo acreditado su merito y aplicacion a la Pintura el Alumno $\mathrm{D}^{\mathrm{n}}$ Felipe Abas, cuyo aprovechamiento es notorio mediante los premios que ha obtenido, y deseando manifestar su aplicación, acordó la Junta, se le nombre Academico Supernumerario» ${ }^{12}$. Cuatro días mas tarde se le oficiaba este nombramiento, según conocemos por el borrador de la carta que se conserva en el archivo académico: «Muy $S^{\mathrm{r}}$ mio: Atendiendo la ${ }^{\text {ta }}$ Particular de esta $\mathrm{R}^{1} \mathrm{Acad}^{\mathrm{a}}$ de $S^{n}$ Luis, a la aplicacion $q^{e}$ V. ha acreditado en la Pintura y a su not ${ }^{\circ}$ aprovecham ${ }^{\text {to }}$ le ha nombrado Academico Supernumerario, esperando esta prueba del aprecio $\mathrm{q}^{\mathrm{e}}$ de V. hace la $\mathrm{Acad}^{\mathrm{a}}$ estimulara más su afición, y $\mathrm{q}^{\mathrm{e}}$ continuara en ella haciendose digno de los demas ascensos. Participolo a $\mathrm{V}$. de acu ${ }^{\text {do }}$, etc. $S^{\mathrm{r}} \mathrm{D}^{\mathrm{n}}$ Felipe Abas» ${ }^{13}$.

La Real Academia de San Luis quería destacar así y honrar con este nombramiento a su antiguo alumno -el primer académico supernumerario del que tenemos constancia en la historia de la Real Corporación-, sin duda pensando los miembros de la corporación en un próximo regreso de Abás a Zaragoza, donde podría incorporarse a las tareas académicas, circunstancia que no se dió. Los Estatutos fundacionales de 1792, tratan en el artículo XVI de los Académicos Supernumerarios en estos términos: «Es mi voluntad, que la Academia pueda poner en esta clase a todos aquellos discípulos, que habiendo obtenido los premios, o dado otras pruebas de habilidad, no se hallen todavía con la consumada perfeccion, que requiere el grado y egercicio de Maestro;

\footnotetext{
11 Ossorio, 1884, p. 2

${ }^{12}$ Archivo de la Real Academia de Nobles y Bellas Artes de San Luis de Zaragoza. Libro de Resoluciones de la Junta Particular de la Real Academia de San Luis, 1793-1817, fol. 109 r.

${ }^{13}$ Legajo de 1805.
} 
pero que estén próximos a merecerlo. Deberán asistir con mas frequencia que otros a los Estudios, tendrán asiento en las Juntas Públicas, y si el Presidente tubiere a bien convocarlos para alguna Junta General, tendrán asiento en ella, y voto consultivo, pero no decisivo».

Lamentablemente no conocemos la respuesta de Abás a la Real de San Luis y la aceptación de este nombramiento, que no dudamos que lo hiciera, pues no existen actas de las juntas posteriores a la del nombramiento ni la carta u otra comunicación de respuesta en el archivo. Debió ser en este momento cuando envió, como agradecimiento a la Real Academia su cuadro de Cristo crucificado actualmente conservado en la colección de la Real Sociedad Económica Aragonesa de Amigos del País.

\section{Abás en la Real Academia de Bellas Artes de San Fernando}

Alcanzado por Abás el primer premio en el concurso celebrado por la Real Academia de Bellas Artes de San Luis de Zaragoza, parecía abrirse ante el joven pintor un brillante porvenir, por lo que pocos meses más tarde, contando casi 21 años de edad, se trasladaba a Madrid para, según recoge Ossorio, «continuar sus estudios bajo la dirección de D. Francisco Goya» ${ }^{14}$. Y así lo encontramos registrado en el Libro de Matricula de la Real Academia de $S^{n}$ Fernando que da principio en $1 .^{\circ}$ de Septiembre de 1795 , y abarca desde esta fecha al mes de abril de 1799, figurando inscrito el día 8 de marzo de 1798, de veinte años de edad, natural de Calaceite e hijo de Manuel y Francisca Aranda ${ }^{15}$.

Concurrió Abás a los premios convocados por la Academia de San Fernando en 1802 y 1805, aunque sin alcanzar el éxito, tal como se pone de manifiesto en el estudio de cada una de estos certámenes.

Premios de 1802. En la Junta ordinaria celebrada por la Real Academia de Bellas Artes de San Fernando el día 6 de diciembre de 1801 los profesores presentaron a la Real Corporación los asuntos asuntos para las pruebas de pensado para los premios correspondientes al año 1802.

Para los premios de pintura correspondientes a la segunda clase firmaron los siguientes opositores: José Alonso del Ribero, Francisco de la Torre, Manuel Sorrentini, José Gómez, José García Pelayo, Miguel Berdejo, Felipe Abás, Angel Palmerani, Antonio del Pozo Robles, Vicente López Naranjo y Angel Arias.

El tema que se debía desarrollar para la prueba de pensado fue el siguiente: La noche subsiguiente a la batalla de Canás, los pocos romanos que habían quedado, estaban tan temerosos y desmayados, que se juntaron en la estancia de Lucio Cecilio Metelo, que era mancebo noble y principal entre ellos, á consultar lo que harían. Y como tenían ya por perdida toda la Italia y todo el gran señorío de Roma con ella, se resolvian á que era mejor pasarse huyendo por el mar a Grecia. Supo P. Corn. Scipion esta tan abatida consulta que en la posada de Metelo se hacía y teniendo por cosa vil apocada, que así se desesperase la nobleza romana del valor de su República y de su gran poderío, con los pocos que le quisieron seguir, se fue á la posada de Metelo, y se puso en medio de los que con el estaban atónitos y despavoridos, que no desampararían la República, antes bien prometieron defenderse de los cartagineses, y seguir á Scipión en todo lo que les mandase ${ }^{16}$.

Para la prueba de repente se fijó la fecha de 4 de julio de 1802, aunque -no sabemos por que razones - no se celebró la Junta esa fecha, sino el día 12 del mismo mes, convocándose a

${ }^{14}$ Ossorio, 1884, p. 2.

${ }_{15}$ Pardo Canalís, 1967, p. 221

${ }^{16}$ Texto extraido de la Crónica General de España de Florián de Ocampo y Ambrosio de Morales, editada por Juan Iñiguez de Lequerica en Alcalá de Henares, 1574-1586, t. II, lib. VI, p. 23. 
los opositores a las nueva de la mañana para sortear entre los mismos las pruebas que debían desarrollar en el espacio de dos horas, mientras que los profesores examinaban los trabajos presentados como prueba de pensado y que cada uno había ejecutado libremente. El tema propuesto fue La lucha de Jacob con el ángel, tema extraido del Génesis: «Quedose Jacob solo, y hasta rayar la aurora estuvo luchando con él un hombre, el cual, viendo que no le podía, le dió un golpe en la articulación del muslo, y se relajó el tendón del muslo de Jacob luchando con él. El hombre dijo a Jacob: «Dejame ya que me vaya, que sale la aurora». Pero Jacob respondió: «No te dejaré ir si no me bendices». El le preguntó: «¿Cual es tu nombre?». «Jacob» contestó este. Y él le dijo: «No te llamarás ya en adelante Jacob, sino Israel, pues has luchado con Dios y con hombres y has vencido». Rogole Jacob: «Dame por favor a conocer tu nombre», pero él le contestó: «¿Para que preguntas por mi nombre?». Y le bendijo allí. Jacob llamó a aquel lugar Panuel, pues dijo: «He visto a Dios cara a cara y ha quedado a salvo mi vida». Salía el sol cuando pasó de Panuel e iba cojeando del muslo. Por eso los hijos de Israel no comen todavía hoy el tendon femoral de la articulación del muslo, por haber sido herido en él Jacob» ${ }^{17}$.

Terminado el ejercicio y tras las consiguientes deliberaciones de los profesores, se concedió el primer premio, consistente a una medalla de oro de una onza a José Alonso del Ribero ${ }^{18}$, obteniendo tres votos cada uno José Gómez, Francisco de la Torre y Felipe Abás, mientras que solo alcanzaron un voto Angel Arias y Angel Palmerani.

Por lo que corresponde al segundo premio, Abás estuvo cerca de alcanzarlo, pues el aragonés obtuvo cuatro votos, el mismo número que Angel Palmerani, mientras que Angel Arias y Francisco de la Torre recibieron cada uno dos votos y José Gómez tres. De acuerdo con el empate entre Abás y Palmerani, decidió la Junta de la Academia llevar a cabo una nueva votación entre ellos, que otorgó el premio — una medalla de plata de ocho onzas- a Palmerani ${ }^{19}$, quien obtuvo tres votos más que Abás.

Premios de 1805. El día 2 de diciembre de 1804 la junta de la Real Academia de Bellas Artes de San Fernando acordaba los asuntos de las pruebas de pensado para los premios de la Academia correspondientes a 1805, publicándose el día 1 de enero de este año el correspondiente cartel de convocatoria firmado por el Secretario, Isidoro Bosarte ${ }^{20}$.

Según este cartel, «La Real Academia de San Fernando propone los acostumbrados veinte premios en diez Medallas de oro y diez de plata a los Profesores de las tres Nobles Artes Pintura, Escultura y Arquitectura, y a los del Grabado. Todos los Opositores que residan en Madrid, han de presentarse al Secretario de la Academia a firmar la Oposición en los primeros quatro meses del presente año: los que no residan en esta Corte, deberán escribirle en el mismo plazo, expresando la facultad y clase en que quieran oponerse. En los últimos quince dias de Junio de este año han de presentar todos los Opositores en la Real Casa de la Academia sus obras concluidas y firmadas, dando aviso al Secretario: en inteligencia de que al que las traxere sin concluir, o viniere pasado el término referido, no se le admitirán, ni tendrá derecho a los Premios; pero los que quieran entregar antes sus obras concluidas, podrán hacerlo».

El tema propuesto por la Academia para la primera clase de pintura fue uno de carácter alegórico-histórico: Carlos III acompañado de la Beneficencia y de la Agricultura, entrega los te-

\footnotetext{
${ }^{17}$ Cap. 32, vv. 24-32.

${ }^{18}$ Natural de Oviedo, de 20 años, nacido en 1782. Además de su participación en los premios de 1802 y 1805, tan solo se conoce de este artista una lámina de Santa Cecilia. Azcárate y otras, 1994, p. 231.

${ }^{19}$ Natural de Jaén, nació en 1785, contando tan solo 17 años. Participó en los concursos de 1802 y 1808 y posteriormente se dedicó a los trabajos escenográficos para los teatros madrileños del Príncipe y de la Cruz. Azcárate y otras, 1994 p. 231.

${ }^{20}$ Uno de estos carteles se conserva en el archivo de la Real Academia de Bellas Artes de San Luis de Zaragoza, en el legajo correspondiente a 1805 . Lo hemos utilizado para la realización de este trabajo.
} 
rrenos de Sierra Morena a Colonos de varias castas para que los pueblen y cultiven. A lo lejos y huyendo del Sol, que presencia esta acción, se ven entre tinieblas ladrones y foragidos que abandonan este sitio. Estableciéndose que «Se pintará este asunto al oleo en un lienzo de dos varas de ancho, y vara y media de alto».

Sobre este tema se ha puesto de manifiesto que «es una alegoría en honor a Carlos III por su labor en materia de política interior, y supone un reconocimiento personal a Campomanes y Olavide por su gestión y por ser de la confianza de su majestad. Por primera vez se propone un tema coetaneo al momento histórico, signo de modernidad y propaganda que paulatinamente marcará las pautas dentro del género de pintura de historia contemporánea frente al medievalismo de los primeros años» ${ }^{21}$.

Se presentaron ocho opositores: Tomás Fernández de Erosa, Miguel Berdejo, José de Odriozola, Francisco Lacoma, Victoriano López, José Alonso del Ribero, Felipe Abás y Angel Arias, y tras la realización del segundo trabajo, la prueba de repente, tal como prescribía la convocatoria: «Entregadas las obras [de pensado] los Opositores de todas profesiones y clases han de hacer nuevas pruebas dentro de la Casa de la Academia en los días que esta señalare, en el término preciso de dos horas, sobre asuntos que se les darán de repente» ${ }^{22}$, que resultó ser Sansón reclinado sobre las faldas de Dalila es aprisionado por los filisteos.

Tras la realización de la obra, se llevó a cabo la votación de los trece vocales existentes en la Junta, recibiendo un voto Miguel Berdejo; dos votos Victoriano López y cinco José Alonso del Ribero, a quien se le concedió el primer premio consistente en una medalla de oro de tres onzas que se le entregaría en sesión ordinaria de 27 de julio. Felipe Abás no tuvo ningún voto a su favor.

Por lo que corresponde a la votación para el segundo premio, Abás y Berdejo recibían un voto cada uno de ellos; López cinco y José Odriozola ${ }^{23}$ seis votos, concediéndose a este la medalla de oro de dos onzas como premio.

\section{Ultimos años de su vida}

Conocemos escasos datos de la vida de Felipe Abás tras abandonar las aulas de San Fernando, posiblemente al concluir el curso académico en junio de 1805. Debió ser por entonces cuando, creado ya académico supernumerario de la Real de Bellas Artes de San Luis de Zaragoza el día 1 de febrero del mismo año, ejecutó y envió a la corporación zaragozana la copia del Cristo Crucificado que Goya había entregado en 1780 a la Real Academia de Bellas Artes de San Fernando como trabajo de recepción como miembro de la misma y que en aquellos años se encontraba en la iglesia del madrileño convento de San Francisco el Grande ${ }^{24}$. Aunque desconocemos la documentación que nos pueda aportar noticias a este respecto, debió contraer matrimonio, del que por lo menos tuvo descendencia femenina, pues Ossorio recoge entre sus obras un retrato de «una de sus hijas».

Felipe Abás fue uno de los perítos que intervinieron en la tasación y partición de los bienes del matrimonio Goya-Bayeu que quedaron, considerándose gananciales, a la muerte de Josefa

\footnotetext{
21 Azcárate y otras, 1994, p. 240.

22 Cartel..., 1805. Ver nota 20.

${ }^{23}$ Nacido en 1782 en Cestona, se matriculó en la Real Academia de Bellas Artes de San Fernando en 12 de febrero de 1796, cuando contaba 14 años. Dedicado a la carrera militar en el arma de Artillería sin descuidar su preparación científica y artística. Años más tarde fue nombrado Académico de mérito. Fue autor de un tratado de matemáticas y de otros de carácter científico. Falleció en 1864. Azcáratey otras, 1994, pp. 239-240.

${ }^{24}$ Sobre este cuadro ver Morales y Marín, 1994, pp. 157-158.
} 
Bayeu el día 20 de junio de 1812, tal como recoge ya Sánchez Cantón en 1946: José García «por lo correspondiente a muebles de casa y ropas», Antonio Martinez, diamantista «por lo que toca a los brillantes, oro y plata» y a Felipe Abás «por lo respectivo a las pinturas» ${ }^{25}$. Los tasadores aceptaron su cargo el 25 de octubre del mismo año, manifestando que lo ejecutarían bien y fielmente, lo que se llevó a cabo, para las pinturas el día 26 del mismo mes de octubre de $1812^{26}$.

Poco mas sabemos de su actividad a no ser su intervención en el lienzo conocido como Alegoría de la Villa de Madrid ejecutado por Francisco de Goya en 1810 por encargo del Ayuntamiento de Madrid y actualmente en el Museo Municipal. Goya pintó una composición alegórica y en un medallón el rostro del monarca José I Bonaparte, destacando el Regidor don Tadeo Bravo de Rivero — quien había hecho el encargo — el talento de Goya que «ha sabido vencer las dificultades que ofrece la ausencia del Rey, y el no haberse proporcionado hasta ahora alguna otra copia que la estampa de medio perfil que, grabada en Roma, tuve el honor de presentar en una de las Juntas Municipales. Con este corto auxilio ha compuesto ya el Sr. Goya un cuadro digno por cierto de todos los objetos a que se dedica...» ${ }^{27}$. Dos años más tarde, al entrar en Madrid el victorioso duque de Wellington el día 12 de agosto de 1812 tras la batalla de Salamanca, se tomaba poco después el acuerdo de que fuera borrado el rostro del soberano francés y se colocase encima del mismo la palabra «Constitución», que poco iba a durar, pues el día 2 de noviembre de ese mismo año 1812 al ocupar nuevamente Madrid las tropas francesas se acordaba en sesión de 30 de diciembre que «se pase oficio a D. Francisco Goya, autor de dicho cuadro, para que inmediatamente concurra a las Casas Consistoriales, y le vuelva a poner en el ser y estado que se hallaba, borrando la palabra Constitución y sustituyendo el rostro del rey José».

Este trabajo se hizo con celeridad pues tres días más tarde, el 2 de enero de 1813 oficiaba Goya al Secretario del Ayuntamiento de Madrid, don Juan Villa y Olier en estos términos: «Puede Usted hacer presente a la municipalidad de la villa de Madrid, que el quadro de la alegoría está ya como en su primitivo tiempo, con el retrato de Su Magestad, el mismo que yo pinté, como quando salió de mis manos» ${ }^{28}$. Por otro documento de Goya, enviado en la misma fecha y al mismo Secretario del Ayuntamiento, sabemos que el trabajo fue ejecutado por Felipe Abás: «Señor Don Juan Villa y Olier. Mi discípulo Don Felipe Abás me ha dicho que diga yo lo que se le debe dar por su trabajo de descubrir el retrato de Su Magestad en el quadro de la alegoria que representa la Villa de Madrid hecho por mi mano, que Vsted lo ha ordenado así, y yo me combengo en hacer lo que Vsted ordena; lo que se le debe dar es, ochenta reales de vellon; según mi parecer es lo justo» ${ }^{29}$.

Algunos meses mas tarde moría en Madrid Felipe Abás, afirmando Ossorio que contaba 36 años de edad, por lo que posiblemente ocurriera en el mes de mayo — había nacido el día 30 de abril de 1777-, afirmando este autor que «dos días antes de ocurrir esta, le había entregado su maestro Goya el título de pintor del Ayuntamiento de Madrid» ${ }^{30}$ todavía gobernado por los franceses.

A la marcha definitiva de la guarnición francesa de Madrid el 27 de mayo de 1813, por orden del Concejo se vuelve a cubrir el rostro del monarca francés y colocar la palabra «Constitución». En esta ocasión, Goya encargaría el trabajo a un discípulo hoy desconocido, Dionisio Gómez, quien el día 23 de junio percibió 60 reales por su trabajo. Con toda seguridad, para esa fecha, Felipe Abás ya había muerto.

\footnotetext{
25 Sánchez Cantón, 1946, p. 80 nota 1.

26 Sobre este tema de la tasación de pinturas ver el interesante y definitivo artículo de Cruz Valdovinos, 1987.

27 Morales y Marín, 1994, p. 301.

28 Morales y Marín, 1994, p. 301.

29 Morales y Marín, 1994, pp. 301-302.

30 Ossorio, 1884, p. 2.
} 


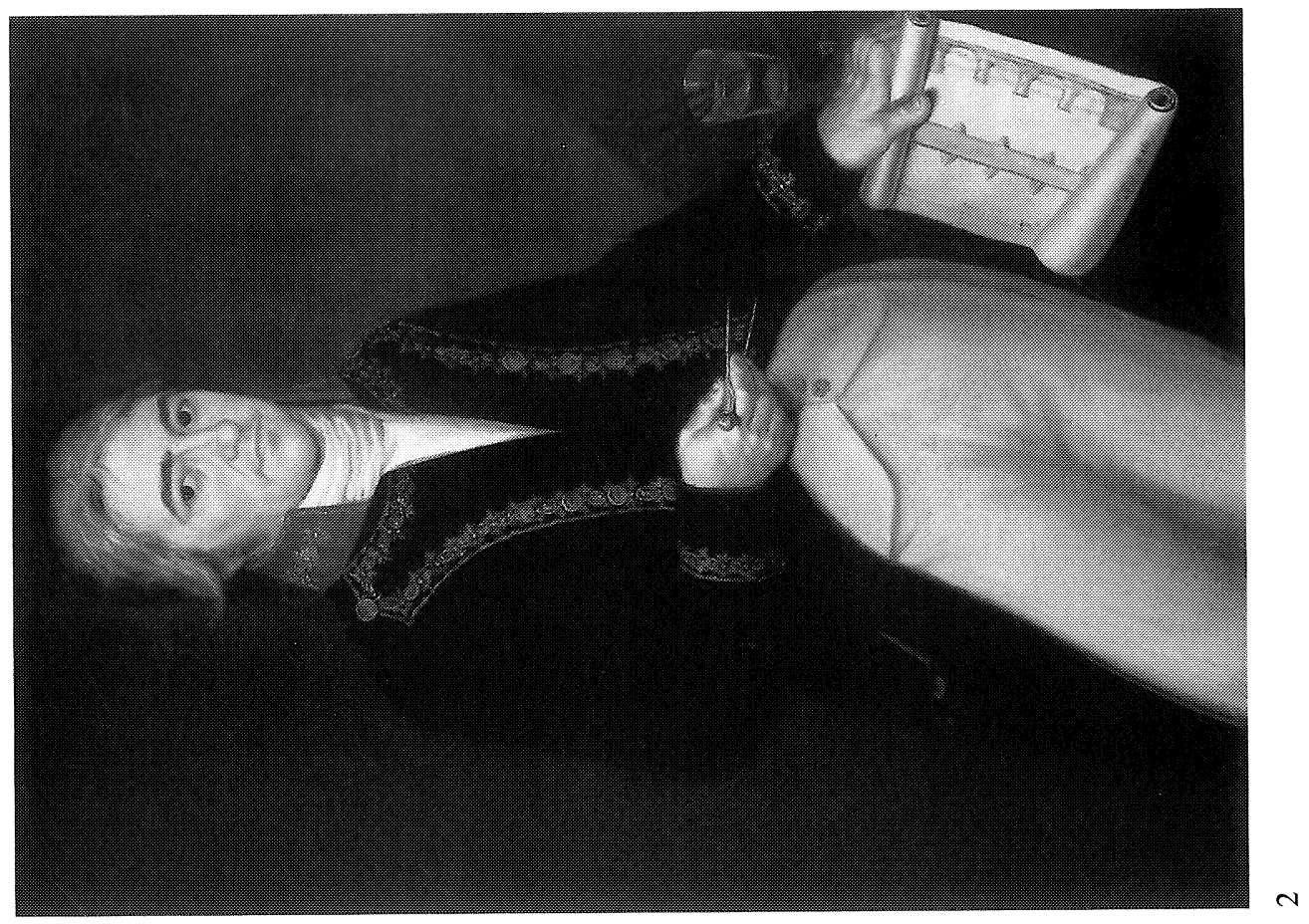

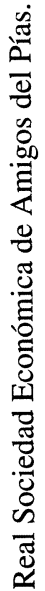

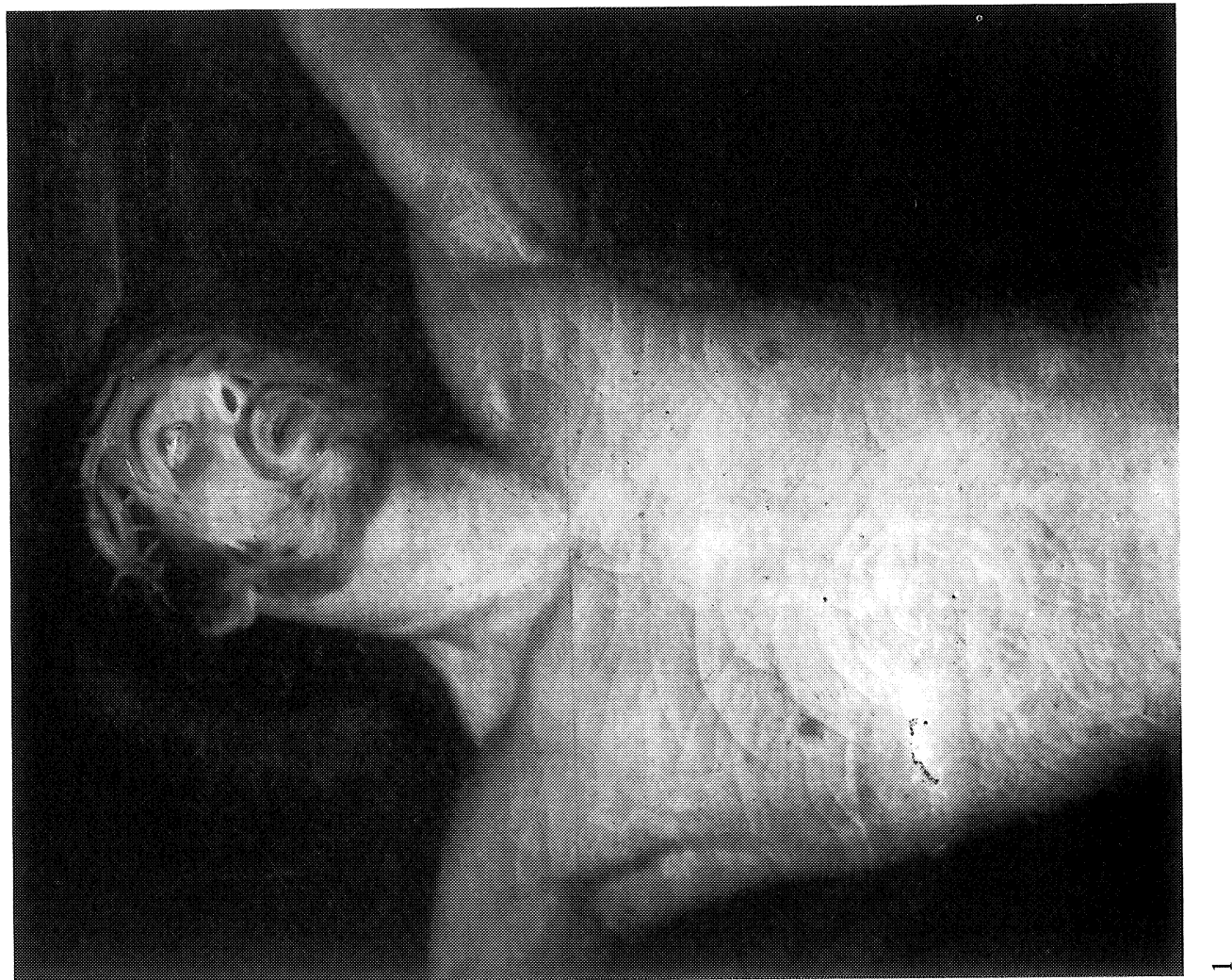

ㅇํㅇ

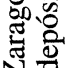

N

宅 \&

ठำ

ช

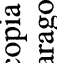

유

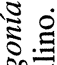

ᄋำ

\&

ธิ

淧泀

Uथ

$-i \mathrm{i}$

览䚾 


\section{Obra}

Ossorio nos proporciona importante información sobre las obras llevadas a cabo por Abás citando, además de los cuadros ya mencionados de El samaritano y la copia del Cristo crucificado de Goya, el «retablo de San José en una iglesia de Aragón, y la colección que su familia conserva y consta de un lienzo que figura el Papa San Gregorio; retratos del principe Fernando, después Rey de España, y de su primera esposa; dos retratos del autor y otro al oleo de una de sus hijas; varias miniaturas, dibujos y retratos, y un boceto representando a Santa Orosia, cuyo cuadro, que destinaba a la iglesia de Calaceite, no llegó a terminar por haberle sorprendido la muerte a la edad de 36 años» ${ }^{31}$.

Nuestras investigaciones en Calaceite, donde todavía residen miembros de la familia Abás, no han dado como resultado el hallazgo de los cuadros citados por Ossorio.

De estas obras destacamos el Cristo Cruficiado, copia de Goya que ya hemos mencionado se encuentra en la actualidad en la colección de la Real Sociedad Económica Aragonesa de Amigos del Pais (Fig. 1). Posible regalo de Abás a la Academia de San Luis con motivo de su nombramiento como académico supernumerario en 1805. Pintado al oleo sobre un lienzo de medidas $265^{\prime} 5$ x 156,5 cms. - igual tamaño que el original - aparece ya con el número 67 en el Catálogo de las pinturas y esculturas que posee y se hallan colocadas en las salas de la Real Academia de Nobles Artes de San Luis de la ciudad de Zaragoza, trabajo que llevaron a cabo en 1828 por los academicos Narciso Lalana y Tomás Llovet, directores de pintura y escultura respectivamente, de la Real Academia ${ }^{32}$, recogiéndolo también Viñaza: «Hay una copia de igual tamaño, hecha por Felipe Abás, que se conserva en la Sociedad Económica Aragonesa de Amigos del Pais» ${ }^{33}$. Es citada esta copia por la mayor parte de los autores que se han ocupado de esta pintura religiosa de Goya ${ }^{34}$.

En la reedición de las Recitaciones de la Historia Política y Eclesiástica de Calaceite de Vidiella, llevada a cabo en 1996, y ya varias veces comentada, se incluye este texto que nos pone de manifiesto la existencia de otra obra de Abás: «D. José Benito Olagorta, secretario del Ayuntamiento de Santander, díceme por carta (1912) poseer una notable obra del pintor Abás, procedente del pueblo de Renedo, en aquella provincia. Es un cuadro de 1,60 metros de alto por 1,20 de ancho, que contiene el retrato de un arquitecto o ingeniero llamado al parecer Mateo del Castillo, según se desprende de un pergamino que la figura sostiene en sus manos con un proyecto de puente firmado con aquel nombre. El cuadro está firmado: Felipe Abás, año 1808. El personaje vese acompañado de esferas, libros y papeles. Dice el Sr. Algorta: «El cuadro es goyesco; de tal manera, que si no estuviera suscrito, se aseguraria que era del Maestro» ${ }^{35}$.

Y vinculado a este retrato - en la actualidad en paradero desconocido - con algunas similitudes por la descripción que conocemos, tenemos que poner un retrato masculino recientemente localizado en una colección particular de Zaragoza y en la actualidad depositado en el Museo de Zaragoza (Fig. 2). Se trata de un oleo sobre lienzo, de medidas 1,05 ×0,77 m., firmado $\ll \mathrm{F}^{\mathrm{e}}$. Abás $\mathrm{P}^{\mathrm{xit}}$ « en el plano (Fig. 3). Representa a un hombre, de tres cuartos - prácticamente hasta la rodilla - vestido con uniforme del cuerpo de ingenieros: casaca azul bordada, con chaleco de color amarillo y pantalón del mismo color. El efigiado aparenta tener entre cua-

\footnotetext{
31 Ossorio, 1884, p. 2.

32 Publicado en el Boletín del Museo Provincial de Bellas Artes, núm. 12, Zaragoza, 1926.

33 Viñaza, 1887, p. 202.

${ }_{34}$ Jeaninne Baticle, en Goya, Grijalbo Mondadori, Barcelona, 1995, p. 263, dice que Abás presentó en 1802 a la Academia de San Fernando una copia del Cristo Crucificado de Goya, preguntándose si será el mismo cuadro que se conserva en el Museo de Santa Cruz de Toledo.

35 Vidiella, edición de 1996, apéndice 259, p. 401.
} 


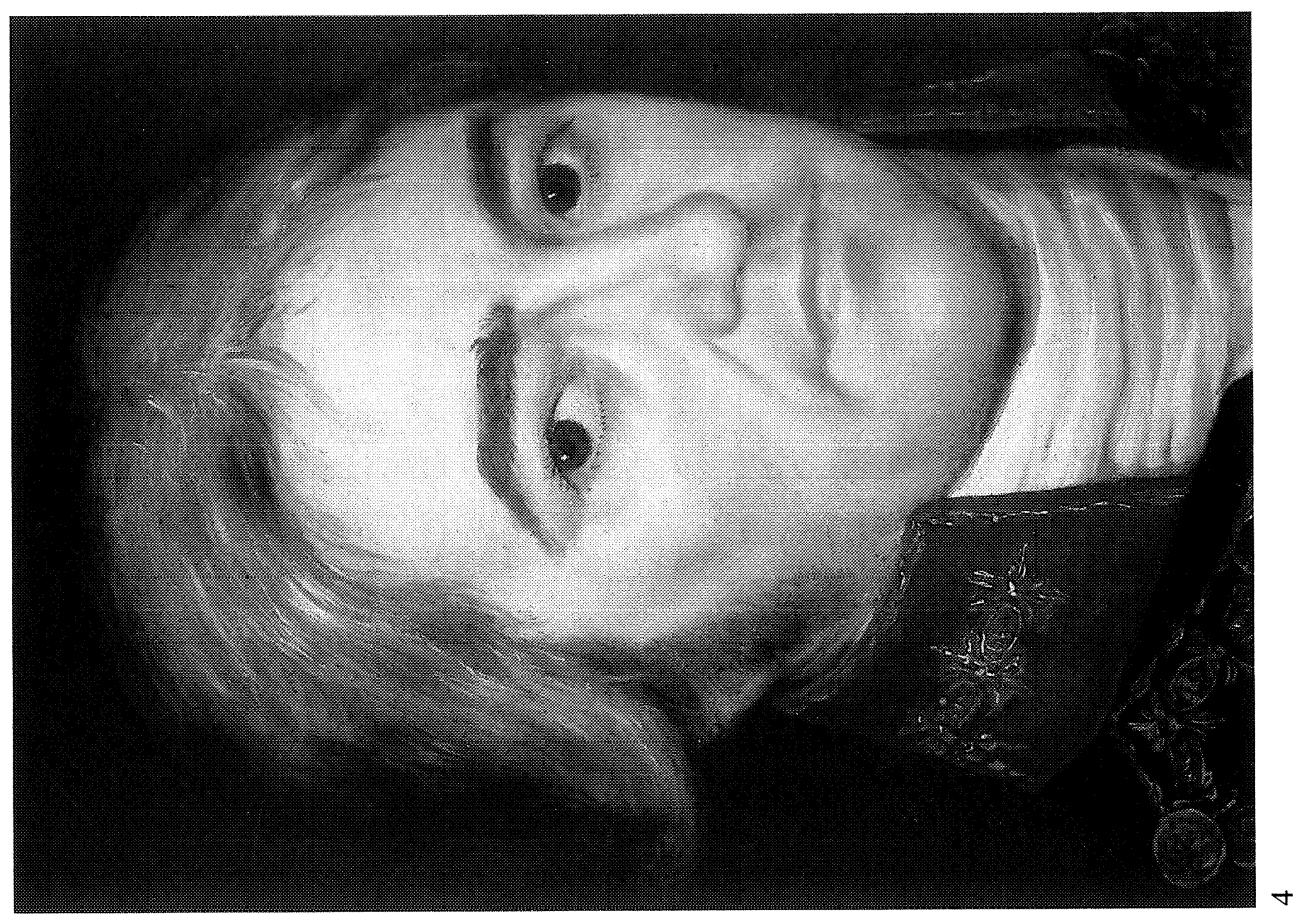

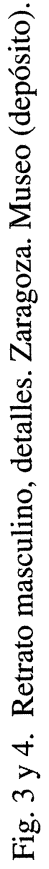


renta y cincuenta años de edad. Apoya la cadera izquierda sobre el borde de la mesa de trabajo de la que es perceptible un ángulo con un borde diagonal en fuga. Sobre la mesa hay un vaso de agua que contiene tres pinceles y junto a él un estuche metálico cerrado con acuarelas que se halla dispuesto para colorear el plano de un puente, con planta y alzado, que sostiene con la mano izquierda. Tiene el brazo derecho apoyado en la cintura y sujeta un compás abierto.

Este cuadro sorprende por la perfección, particularmente de las carnaciones, muy próximas a las calidades conseguidas por su maestro y quizás para su realización se inspirara Abás en el retrato que Goya realiza del Marqués de San Adrián (Museo de Pamplona). Sobre un fondo neutro de color verde grisáceo, sobresale la figura del personaje con una pose quizá algo desafortunada para el retrato, que en cambio consigue resolver positivamente con el dominio de una buena técnica. El uso del color es muy acertado, el frío azul de la casaca en pinceladas finas y estiradas contrasta con el suntuoso bermellón del cuello y con los dorados amarillos pastosos en los entorchados y pinceladas más finas en el propio borde del chaleco y pantalón, destacando el blanco cremoso que aflora entre el rojo, el amarillo y el azul. Pero es sin duda, en la resolución de la cabeza, donde la feliz inspiración, ha conseguido acercarse a la maestría del propio Goya. El pelo entrecano, algo descuidado, y las cejas pobladas, tratados con pinceladas sueltas y rápidas, dotan al rostro de fuerza y movimiento, en contraste con el tratamiento de las carnaciones que se resuelve superponiendo pinceladas finas de colores cálidos, con una técnica depurada, donde luces y sombras juegan un papel esencial en la volumetría del rostro. Este retrato ejecutado por Felipe Abás, muestra al espectador la imagen de un hombre frío, de escrutadora mirada y de gran firmeza de carácter (Fig. 4).

\section{BIBLIOGRAFIA}

AzCÁRATE y otras: Historia y Alegoría: Los concursos de pintura de la Real Academia de Bellas Artes de San Fernando (1753-1808), Real Academia de Bellas Artes de San Fernando, Madrid, 1994.

CRUZ VALdovinos, José Manuel: «La partición de bienes entre Francisco y Javier Goya a la muerte de Josefa Bayeu y otras cuestiones», Goya Nuevas Visiones. Homenaje a Enrique Lafuente Ferrari, Amigos del Museo del Prado, Madrid, 1987, pp. 133-153.

Morales y Marín, José Luis: Goya. Catálogo de la pintura, Real Academia de Nobles y Bellas Artes de San Luis, Zaragoza, 1994.

OSSORIO Y BERNARD, Manuel: Galería biográfica de artistas españoles del siglo XIX, Madrid, 1884.

PaRdo CANalís, Enrique: Los registros de matrícula de la Academia de San Fernando de 1752 a 1815, CSIC, Madrid, 1967.

SÁnchez CANTón, Francisco Javier: «Como vivía Goya», Archivo Español de Arte, 1946, pp. 73-109.

VIDIELla, Santiago: Recitaciones de la Historia Política y Eclesiástica de Calaceite, reedición, Ayuntamiento de Calaceite, Instituto de Estudios Turolenses y Centro de Estudios Bajoaragoneses, 1996. 